\title{
COMMUNICÁTIONS
}

\section{ANATOMICAL STUDIES OF THE TRABECULAR MESHWORK OF THE NORMAL HUMAN EYE*}

BY

\author{
NORMAN ASHTON, ALFRED BRINI, AND REDMOND SMITH \\ Department of Pathology, Institute of Ophthalmology, University of London
}

IN recent years increasing evidence has been obtained of an abnormal resistance to aqueous outflow in eyes affected with chronic simple glaucoma; it now appears essential to determine to what extent this may be attributable to structural changes and at which particular site or sites in the drainage area the impediment is located. In this problem, consideration of the trabecular meshwork is clearly of primary importance, but a true assessment of morbid anatomical changes and their distinction from appearances due to artefact can be based only upon an accurate knowledge of normal structure. Although many anatomical studies of the trabecular meshwork are available both in papers and textbooks, they are frequently deficient in detail and differ from each other in important respects. As a preliminary to future work on pathological material, we have therefore thought it necessary to summarize existing knowledge and to carry out, by old and new methods, a further anatomical study of this region.

\section{REVIEW OF LITERATURE}

\section{(1) Limits AND General Shape}

(a) Microscopical Appearances.-The first classical descriptions of the meshwork of the chamber angle as revealed in sections and flat preparations are to be found in the works of Rochon-Duvigneaud (1892; 1893), Asayama (1901), Virchow (1910), and Salzmann (1912). These authors all give references to earlier communications on the subject, but they are of historical interest only and will not be discussed here.

According to Salzmann (1912), the meshwork forms a three-sided prismatic band; anteriorly the edge is extremely sharp and unites with Descemet's membrane and the adjacent corneal lamellae; posteriorly trabeculae extend to unite with the scleral spur, the anterior surface of the ciliary body, and indirectly with the root of the iris; the outer surface borders directly upon corneal and scleral tissue and upon the inner wall of Schlemm's canal or the loose tissue surrounding it; the inner surface is free and is in contact with the aqueous humour of the anterior chamber.

*Received for publication February 28, 1956. 
In an earlier study, Virchow (1910) had considered this meshwork to consist of two main parts:

(i) Scleral Meshwork, or corneo-scleral trabeculae, which is the outer portion connected with the scleral spur;

(ii) Uveal Meshwork, or rudimentary pectinate ligament, which is the inner portion terminating in the ciliary body and iris root.

This description is now generally accepted.

In a recent study, Burian, Braley, and Allen (1954), while agreeing with this subdivision, described three groups of fibres in the uveal meshwork, according to their respective connexion with the meridional fibres of the ciliary muscle, its circular fibres, and the iris root.

(b) Gonioscopic Appearances.-These have been described by Busacca (1945), François (1948), Troncoso (1947), and Burian and others (1954).

Gonioscopic terminology does not correspond to histological terminology, since the gonioscopic view only takes in the inner surface of the structure concerned, whereas histological terms refer to the appearances of the tissues in section.

The trabecular band as seen gonioscopically is that area of the meshwork which appears to lie between Schwalbe's line and the white band of the scleral spur. Histologically, this corresponds to the scleral part of the meshwork plus that part of the uveal meshwork and those fibres of the pectinate ligament which are situated between the scleral spur and Schwalbe's line.

(i) Trabecular Band.-This forms, between Schwalbe's ring and the scleral spur, a circular band of grey-white colour, fairly bright in young people but more matt and pigmented in older subjects. Usually the anterior part of the trabeculae is whiter; while the posterior part, where Schlemm's canal lies, is greyish. Deposits of pigment when present are more prominent on Schwalbe's ring and at the level of Schlemm's canal. In favourable cases Bangerter and Goldmann (1941) saw a fine superficial striation on the trabeculae, and Busacca (1945) asserts that a surface examination with a narrow slit shows numerous little cavities corresponding to the interstices of the meshwork. Trantas (1928) found vessels in the normal trabeculae whereas François (1948) had never observed them.

(ii) Ciliary Band.-Seen gonioscopically, this consists of the anterior extremity of the ciliary body, as observed through the posterior part of the uveal meshwork, and of the rudimentary pectinate ligament.

\section{(2) Shape AND ORIENTATION OF Fibres}

Whereas the shape, limits, and subdivisions of the trabeculae have in general been accepted by all authors, two different opinions have been expressed concerning the shape and orientation of the fibres themselves.

(a) Scleral Meshwork.-Here most authors consider the fibrils to consist of flat anastomosing bands of tissue lying in several superimposed planes, each parallel to the surface of the cornea, and inter-connected by scattered oblique anastomoses. Owing to the triangular shape of an antero-posterior section of the meshwork, only two or three trabecular layers are seen close to Descemet's membrane, whereas fifteen to twenty are present near the scleral spur. Rochon-Duvigneaud (1893) described the proximal part of each layer as resembling a perforated plaque. Asayama (1901) agreed with this description; it is of interest to note that he suggested the possible value of studying and comparing the permeability of the meshwork 
in young and old eyes. The description of Salzmann (1912) is similar to that of Rochon-Duvigneaud (1893).

These three authors, by studying antero-posterior sections and flat preparations, came to the conclusion that the flat bands of tissue in the scleral meshwork are predominantly circular in disposition, that is to say parallel to Schlemm's canal. While many authors agree with this description, later works tend to imply that the meshwork is formed of antero-posterior fibres. Thus Redslob (1939), quoting De Lieto Vollaro (1902), writes:

La structure histologique de la partie sclérale du système trabeculaire est formeé par des séries radiales de petites baguettes, plus ou moins régulières . . .

François (1948) quoted Redslob's description, and the text of Wolff's “Anatomy of the Eye and Orbit" (1954) suggests that he also shared this view.

Thus in classical textbooks may be found two quite different opinions concerning the shape and orientation of the scleral trabeculae, some authors describing circularly disposed bands and others little antero-posterior rods.

(b) Uveal Meshwork.-In its outer layers its constitution is quite similar to that of the corneo-scleral trabeculae. In the innermost layer, on the other hand, the fibres are radially disposed and connected with the anterior surface of the iris or the iris root. They are rather circular in cross section and form a very loose and irregular meshwork, which probably represents a vestige of the pectinate fibres, with which, in lower animals, one associates the spaces of Fontana.

\section{(3) Histological Constitution of Each Fibre}

Again we are indebted to Salzmann (1912) for an accurate description of the histological constitution of each fibre of the meshwork.

(a) Scleral Meshwork.-After van Gieson and orcein staining, four successive concentric layers are visible which, from within outwards, are as follows:

(i) A thick central core of collagen fibrils running parallel to the long axis of the meshwork fibres.

(ii) A layer of strong elastic fibres, having the same orientation and, consequently, appearing in the form of dots in antero-posterior section.

(iii) A glass membrane resembling Descemet's membrane, of which it is probably a continuation since it has the same morphological and staining characteristics.

(iv) A final endothelial layer which is a prolongation of the corneal endothelium.

While most modern authors have accepted this histological description, not all workers are in agreement, for some claim that it is not possible to recognize the four layers in sections of the trabeculae. Thus Eisler (1930) says that the endothelium and the hyaline membrane are one and the same layer, forming a sponge-like syncytium. Other histologists have found additional elastic fibres inside the connective core, and consequently do not accept the idea of a true elastic layer. While Salzmann (1912) and Teulières and Beauvieux (1930) consider that Descemet's membrane extends into the meshwork to form the glass membrane, Asayama (1901), Virchow (1910), and Eisler (1930) share the opinion that Descemet's membrane terminates at the point where the trabeculae begin.

(b) Uveal Meshwork.-Its constitution is similar, but the individual fibres are circular in cross section and contain no elastin. 


\section{(4) Dimensions of Trabeculae}

Measurements of the thickness of the bands in the meshwork have been made infrequently, either because little importance has been attached to the structural details of this area, or because of difficulty in effecting accurate measurements owing to the great irregularity of the fibres-a feature clearly described by Lauber (1936).

Henderson (1908) states that in children the thickness of the fibres is about $3 \mu$ while in adults of the fifth decade it is 5-7 $\mu$. This increase is due, in his opinion, to sclerosis of the inner collagenous core, which becomes thicker, whereas the surrounding glass membrane becomes thinner. Since the whole thickness of the meshwork remains the same at different ages, Henderson concludes that the inter-trabecular spaces must be reduced with advancing age, and in his view this is the cause of primary glaucoma.

\section{(5) Passage from Anterior Chamber to Schlemm's Canal}

Although our knowledge of the outflow channels of Schlemm's canal has improved considerably in recent years, more limited progress has been made in determining the precise route of the aqueous humour through the trabeculae, and the important question whether open communications exist between the anterior chamber and the canal is still a matter of lively debate. Studies have been performed by purely histological methods, by injections followed by microscopical examination, by reconstruction from serial sections, by the intracameral injection of particles of varying size and of radioopaque materials, and by gonioscopy.

(a) Purely Histological Studies.-Conventional studies with varying staining methods have led to contradictory conclusions which may be grouped as follows:

(i) The opinion that free communications exist between Schlemm's canal and the anterior chamber through the meshwork has been defended by Brücke (1847), Iwanoff and Rollett (1869), Schwalbe (1870), Waldeyer (1874), Asayama (1901), Henderson (1908), Sondermann (1930), Dvorak-Theobald (1934, 1955), Swindle (1937), and Loewenstein (1951).

The last three authors agree with Sondermann, who gave the first detailed account of the open channels through the trabecular meshwork, which are sometimes referred to as Sondermann's internal canals. According to his description these channels are lined with endothelium and may be found in most cases where serial sections have been made. They start as a gap in the inner wall of Schlemm's canal towards its posterior extremity and, leaving the canal at right angles, soon become parallel to the trabecular lamellae and open into the spaces between them.

It is interesting to recall that these openings had been noticed by RochonDuvigneaud (1893):

Petits prolongements coniques de la lumière du canal, qui penètrent dans les mailles du réseau et semblent s'ouvrir au milieu d'elles.

But he considered histological studies less reliable than his injection methods, and this led him to conclude that free communications between the trabeculae and Schlemm's canal did not in fact exist.

(ii) Many authors have concluded from histological evidence that the aqueous humour must pass through an endothelial membrane to gain access to Schlemm's 
canal, and the majority believe that this membrane is the endothelial lining of the canal (Rochon-Duvigneaud, 1893; Leber, 1903; Virchow, 1910; Salzmann, 1912; Maggiore, 1917; Teulières and Beauvieux, 1930; Eisler 1930; Lauber, 1936; Redslob, 1939; Troncoso, 1947; Sugar, 1951).

As a result of his claim that arteries opened directly into Schlemm's canal, Friedenwald (1936) inferred that open channels to the anterior chamber could not exist; because otherwise plasma would necessarily leak into the anterior chamber, raise the protein concentration therein and so interfere with the drainage mechanism. Although he admitted the presence of Sondermann's canals, he believed them to be blind pockets separated from the inter-trabecular spaces by an endothelial layer.

The most convincing evidence of the existence of open communications between the canal and anterior chamber is to be found in the recently published work of Dvorak-Theobald (1955). ' By means of superimposed drawings on transparent paper she was able to trace, through four to six sections, continuous tortuous pathways from the anterior chamber, through the intra-trabecular spaces and into the canal of Schlemm. The figures illustrating this careful work are excellent and now leave little room to doubt the existence of these much-disputed canals.

(b) Injection Methods, with and without Histological Study.-The controversy whether the process of aqueous elimination is one of seepage or of direct flow through the trabeculae dates from the early experiments of Schwalbe (1870) and Leber (1895), who used intracameral injections of carmine and Berlin blue, with such contradictory results that no final conclusion was possible. These studies are now only of historical importance, and those interested in this aspect may find all the references in the bibliography of Maggiore (1917). At a later period, Swindle (1937) found Sondermann's canals on section and confirmed his findings by Indian ink injection into the anterior chamber.

Recent experiments to determine the pore size in the trabecular meshwork have been carried out by Huggert $(1954,1955)$. Suspensions of chromium phosphate and of different bacteria were injected into the anterior chamber of rabbits and human eyes, and the size of particles recovered from the episcleral vessels was measured. In human eyes he found that particles of $1.2 \mu$ and $1.5 \mu$ passed readily through the meshwork; the largest particles able to pass were $4.4 \mu$ in size.

A similar investigation was carried out by François, Neetens, and Collette (1955), who injected the anterior chamber of monkeys and human eyes with Thorotrast (particle size $0 \cdot 1 \mu$ ) and Angiopac (particle size 1, 2, and $3 \mu$ ) under a pressure of $25 \mathrm{~mm}$. Hg. The eyes were then fixed and the internal layers of the trabeculae removed and examined by micro-radiography. Minute holes were found in the inner wall of Schlemm's canal, and these appeared as white holes on the microradiogram. Thorotrast penetrated freely and immediately, but Angiopac of $1 \mu$ passed less readily, and Angiopac of 2 or $3 \mu$ did not penetrate at all. It was concluded therefore that the trabeculae are traversed by open channels having a size of less than $2 \mu$.

(c) Gonioscopic Examination.-This frequently shows the presence of blood in Schlemm's canal and occasionally it had been seen to enter the anterior chamber when the episcleral venous pressure is increased. Minute streams of blood entering the canal have been observed by Kronfeld, McGarry, and Smith (1942), Hobbs (1950), and Loewenstein (1951). According to Duke-Elder (1955) such streams are most frequently seen at about the 12 o'clock meridian, and since on subsequent examination they tend to occur at the same site it is more reasonable to suppose that 
they enter through pre-existing pores rather than through mechanical ruptures of the trabeculae. On the other hand van Beuningen (1949) and Huggert (1955) have never seen red blood cells enter the anterior chamber.

(6) Nerves in the Trabecular Meshwork

Kolmer (1914) demonstrated the presence of nerve fibres in the trabeculac by the Bielschowsky-Agduhr method. He found them on serial sections cut parallel to the surface of the iris, but he could not find them in the meshwork itself and, therefore, suggested that these nerves cross the anterior chamber angle and end elsewhere.

More recently, Vrabec (1954) applied a modified Bielschowsky method to human eyes and found many nerve fibres in the trabeculae. Since on anteroposterior section of the eye these fibres are cut in cross-section they are not easily seen. Vrabec also cut tangential sections parallel to the surface of the limbus, and sections in the same region which were perpendicular to the surface of the eye. In these preparations he was able to show that both myelinated and non-myelinated nerve fibres with small endings in the trabecular meshwork, exist in the human eye.

\section{Present Study}

(1) MATERIAL.-This study is based upon the microscopical examination of post-mortem eyes and eyes removed at operation. The best material for a study of normal anatomy would, of course, be fresh post-mortem material, but this, unfortunately, is very seldom available. Teng, Paton, and Katzin (1955) used eyes from a bank, which were consequently preserved without fixation at low temperatures, sometimes for several days. The eyes we obtained were removed 12 to 24 hours after death and fixed immediately; nevertheless, earlier fixation is certainly preferable, and we therefore studied in addition a series of eyes removed for melanomata which had been placed in fixative immediately after enucleation in the operating theatre. In such specimens care was taken to exclude all those in which the tumour, or detached malignant cells, had encroached upon the anterior segment, and all eyes showing inflammatory changes in the filtration angles.

\section{(2) METHODS}

(a) Histological Techniques.-Most specimens were fixed in 10 per cent. formol saline; some were fixed in a modified Carnoy solution (methyl alcohol $45 \mathrm{ml}$., ethyl alcohol $45 \mathrm{ml}$., glacial acetic acid $10 \mathrm{ml}$.) or basic lead acetate 4 per cent., or ethyl alcohol 80 per cent., but no striking differences were noted between the results from various methods.

The eyes with choroidal melanomata were embedded in celloidin. Those removed post mortem were prepared for study by removing the posterior half of the globe with a razor blade, discarding the lens and vitreous, and dividing the anterior segment into several approximately triangular fragments by a series of radial incisions from the centre of the cornea. After 
fixation the pieces were embedded in paraffin wax and cut at $8 \mu$, or in celloidin and cut at $12 \mu$.

Celloidin embedding appears to preserve the spatial relationship of the corneal, scleral, and trabecular fibres, but the sections are necessarily thick. Paraffin sections are thinner but show marked distortion and separation of the tissue planes. Both methods were therefore used in this work according to our special aim in each case.

Three different planes of section were chosen in order to obtain a clear view of the topography of the meshwork:

(i) Antero-posterior Sections.-To avoid a possible oblique incidence, these were made to pass through the pupil.

(ii) Tilted Frontal Sections.-The plane of these was perpendicular to the minute rods seen in the meshwork on antero-posterior section, that is to say, perpendicular to the corneal surface in the limbal region.

(iii) Flat Sections.-The plane of these was parallel to the limbal surface and they were thus similar to those made by Teulières and Beauvieux(1930) and formerly by Virchow (1910) in the Macacus (Fig. 1).

The majority of sections from eyes with choroidal melanomata were stained with haematoxylin and eosin; somewere stained for elastic tissue, which appeared to be demonstrated equally well by means of Weigert's resorcin-fuchsin, orcein, or Verhoeff's technique. The various layers inside the fibres of the meshwork were readily visible with Masson's trichrome and Verhoeff's elastic stain.

(b) Measurements.-An attempt was made to measure the thickness of the trabeculae, the spaces between them, and the pores or gaps in individual planes of the trabeculae. For this purpose a

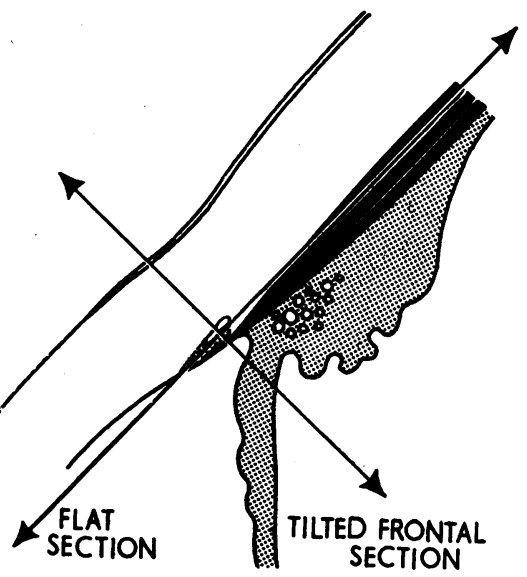

Fig. 1.-Planes of tilted frontal sections and flat sections. micrometer, calibrated against a stage micrometer of known dimensions, was inserted in one of the eye-pieces of a binocular microscope (magnification $\times 950$ ). Measurements were attempted only in sections taken in the conventional antero-posterior plane.

(c) Flat Preparations.-These were made from the fresh material. The anterior segment having been separated as previously described, the uveal tract was removed so that the trabecular meshwork could be inspected under the dissecting microscope and a portion removed with fine forceps. One limb of the forceps was inserted into Schlemm's canal, the blade was closed, and a portion of the trabecular tissue was pulled away from the globe. This manoeuvre usually resulted in a clean tear at the posterior attachment of the 
meshwork at the scleral spur, whereas the anterior attachment usually remained adherent and a portion of Descemet's membrane and corneal endothelium came away with the removed specimen (Fig. 2).

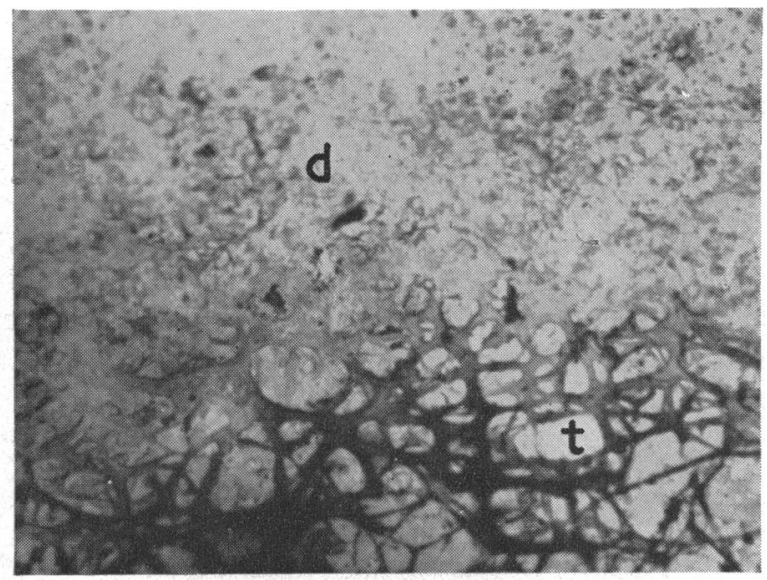

FIG. 2.-Photomicrograph of flat preparation made by pulling the trabeculae forward. Note how the corneal endothelium, with Descemet's membrane $(d)$ remains adherent to the anterior extremity of the trabeculae $(t): \quad \times 300$.

Another method consisted of making with a small knife two parallel incisions on each side of the inner wall of Schlemm's canal and close to its edges. It was thus possible to remove that part of the meshwork between the two incisions.

These flat preparations were examined under the conventional microscope after staining with polychrome methylene blue. In their central part they proved to be too thick to allow an entirely satisfactory microscopical examination, but the general state of the meshwork and the orientation of the fibres were clearly visible. More detailed observations could be carried out on the edges of the preparations where it was thinner, or in teased specimens. Several flat preparations were examined with the particular intention of seeking stomata in the outer layers of the trabeculae which form the surface of the inner wall of the canal.

\section{(1) Histological Study}

\section{FINDINGS}

(a) Limits and General Shape.-The present work has not produced any evidence at variance with the classical descriptions of the limits and general shape of the meshwork, and we have nothing of importance to add to the accurate anatomical description given recently by Burian and others (1954). These workers, however, subdivide the uveal meshwork into three components according to the connexion of the fibres with the meridional muscle bundles, with the circular muscle bundles of the ciliary body, or with the iris root: but, in our experience, the ciliary muscle bundles are so frequently ill-defined that it is not always possible to differentiate the particular muscle group to which the uveal trabeculae are attached. This subdivision, therefore, appears 
too indefinite to be of value, and we prefer to consider the uveal meshwork to consist of two main parts:

(i) An Outer Component.-This extends from its anterior attachment at Descemet's membrane and adjacent corneal layers, to fuse, after passing beneath the scleral trabeculae and scleral spur, with the inter-cellular substance of the ciliary muscle.

(ii) An Inner Component.-This consists of a loose irregular reticulum (Fig. 3) and has a similar anterior attachment, but curves around the anterior chamber to merge with the anterior surface of the iris root.

Fig. 3.-Photomicrograph of flat preparation of inner (uveal) part of trabeculae. $\times 1300$.

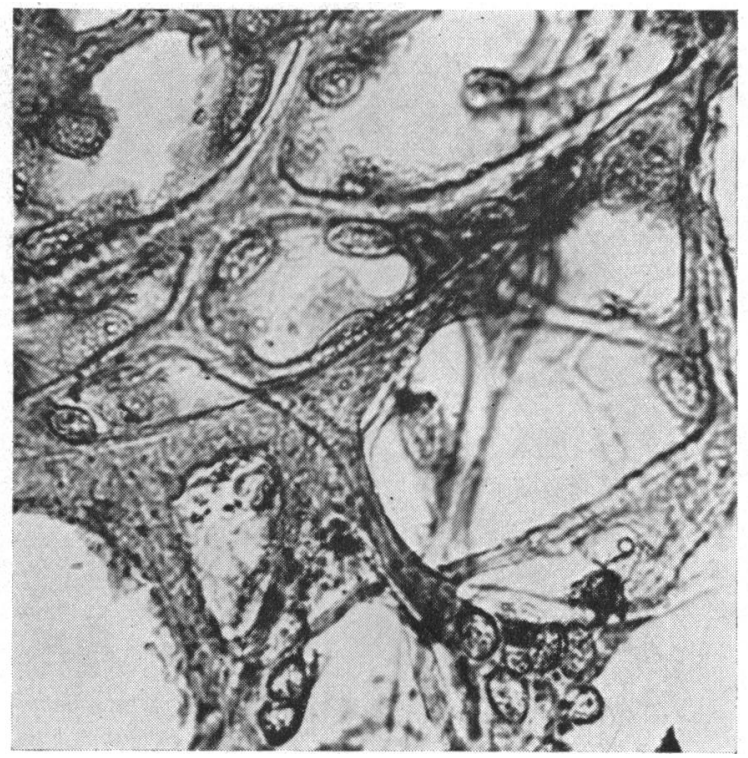

(b) Shape and Orientation of Fibres.-The morphological appearances of the trabeculae naturally vary with the angle of observation. Their main features, as seen in sections of three different planes and in flat preparations, are as follows:

(i) Antero-posterior Sections.-These show minute rods arranged in layers, which are few in number in the thin anterior wedge of the prismatic trabecular band, but become more numerous as the meshwork widens, so that fifteen to twenty layers may be seen at the posterior limit. In their antero-posterior axis the rods are separated by small gaps corresponding to lacunae in the lamellae; these spaces do not lie immediately over one another but are bounded on either side by the adjacent lamellae (Fig. 4, overleaf).

(ii) Tilted Frontal Sections. - These show long fibres parallel with each other and to Schlemm's canal; scattered oblique fibres interconnect the layers. In these sections the individual trabeculae appear much longer than those seen in antero-posterior sections, which indicates that the fibres of the meshwork are predominantly disposed in a circumferential direction (Fig. 5 , overleaf). Hence the appearance of short rods on antero-posterior section is due to the fact 


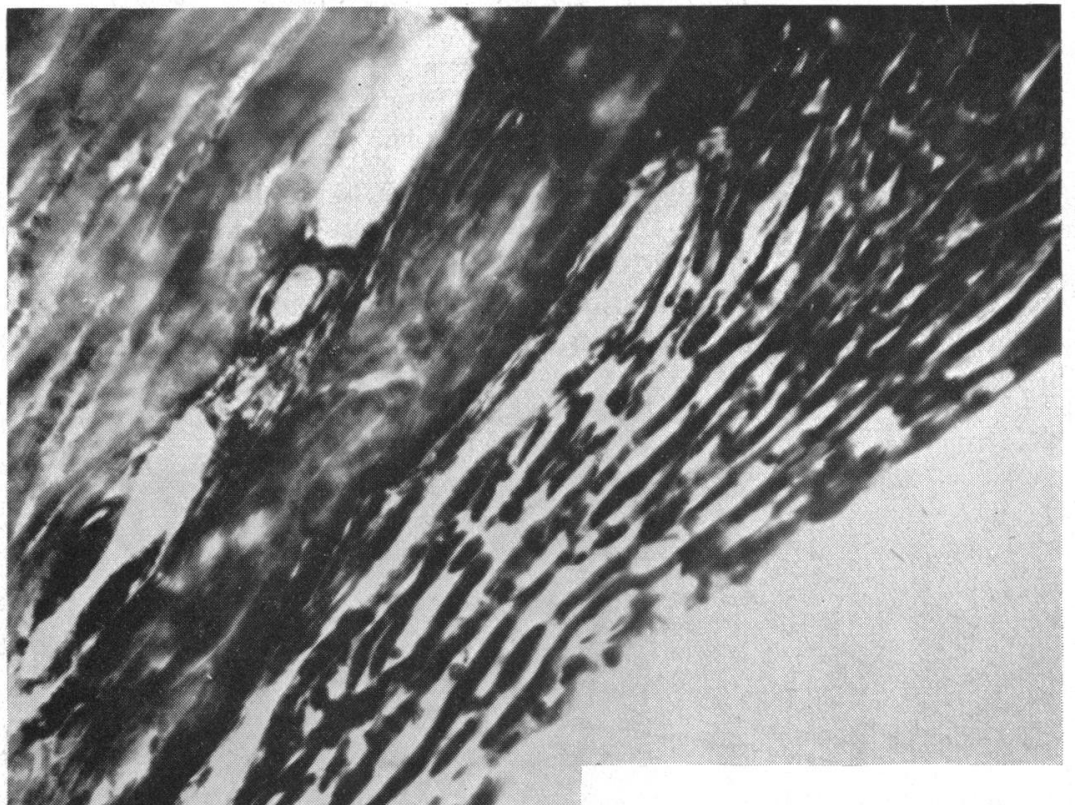

Fig. 5.-Tilted frontal section of trabeculae $(t)$ and Schlemm's canal $(s)$. Note that the individual trabeculae appear longer than in antero-posterior section. $\times 213$.

Fig. 4.-Antero-posterior section of trabeculae and Schlemm's canal, showing that the trabecular pores are covered on either side by the adjacent lamellae. $\times 360$.

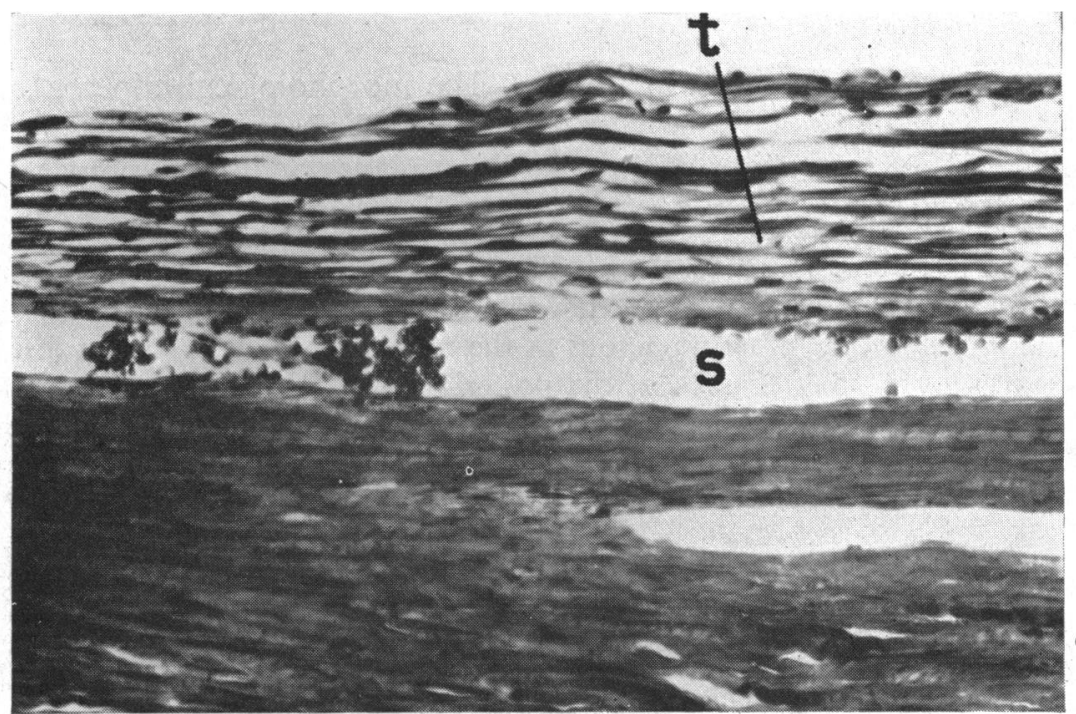


that the cut ends of the broad flattened circumferential fibres are being observed.

In brief, the tilted frontal plane gives longitudinal sections of the trabecular fibres, the antero-posterior plane gives cross-sections of the same fibres.

(iii) Flat Sections Tangential to the Corneo-scleral Surface.-These must be cut serially as only a few pass through the meshwork, and, since it is curved, only one or two of them will actually be parallel to its surface. Provided that the best sections are chosen, it may be seen that the trabecular meshwork is formed by flat fibrous bands which freely anastomose and in so doing form oval lacunae with their longest diameter lying in the circumferential meridian of the limbus, that is to say, parallel to the anterior edge of the scleral spur (Fig. 6).

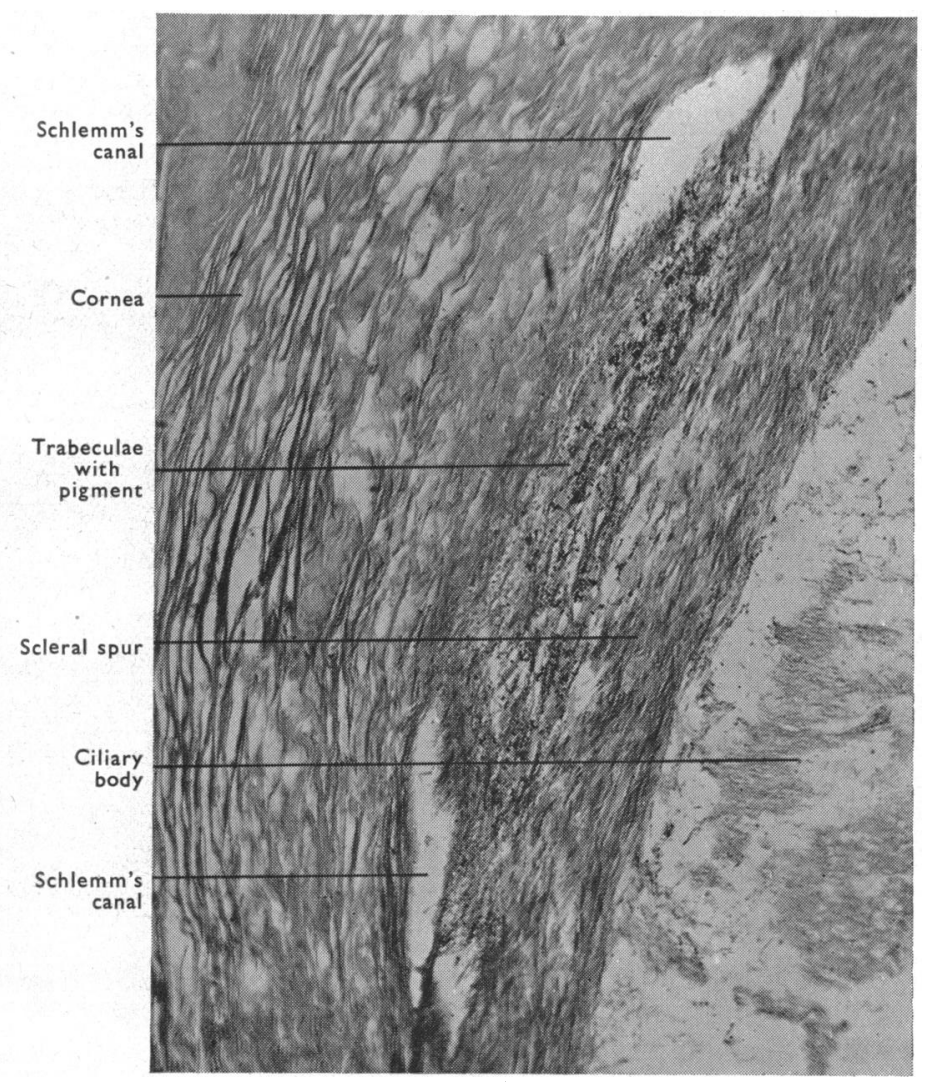

Fig. 6.-Flat section of trabeculae and Schlemm's canal. $\times 112$.

(iv) Flat Preparations.-These show a similar appearance despite the difficulty in seeing through the whole thickness of the meshwork with the microscope. If the edge of the specimen is studied, it will be seen that the trabecular meshwork is formed of layer upon layer of sheets of tissue showing numerous oval lacunae and containing large numbers of fine fibrils running mainly circumferentially. The arrangement is illustrated in Figs 7 and 8 (overleaf); 


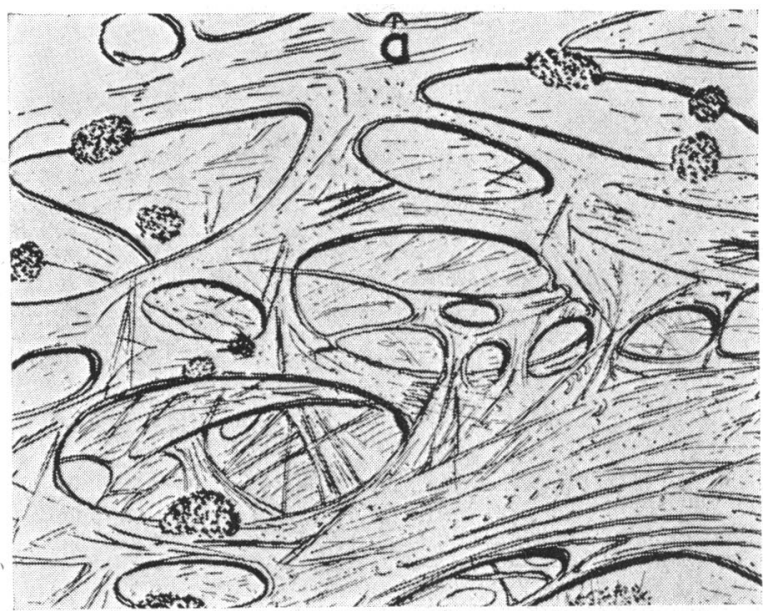

Fig. 7.-Drawing of flat preparation of trabeculae $(a=$ anterior).

FIG. 8.-Photomicrograph of flat preparation of trabeculae. $\times 1125$ :

although the general impression.is of a meshwork composed of communicating bands of tissue, each plane could equally well be said to consist of perforated plaques, the holes in which, being oval, give the impression that the

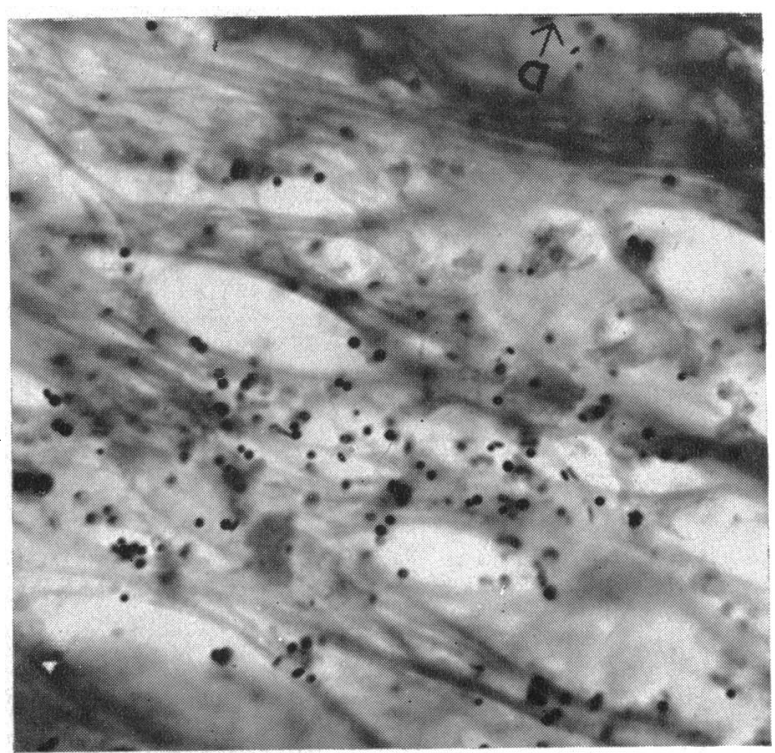
boundary tissue is arranged in bands. Indeed, this band-like structure is less well marked anteriorly, where the holes are rounded or irregular, than posteriorly, where they are predominantly oval.

In the trabecular layer forming the inner wall of Schlemm's canal, these oval lacunae may be seen in flat preparations as irregularly arranged stomata opening directly into the canal itself (Figs 9 and 10, opposite).

(c) Histological Constitution of Each Fibre.-In this regard our findings (Fig. 11, overleaf) closely approximate to those of Salzmann (1912).

(i) Antero-posterior Sections, stained with Verhoeff's elastic stain combined with Masson's trichrome stain, clearly show, on ordinary microscopic examination, four concentric layers from without inwards: endothelial layer, glass membrane, elastic fibres, inner connective tissue core (Fig. 12, overleaf). 


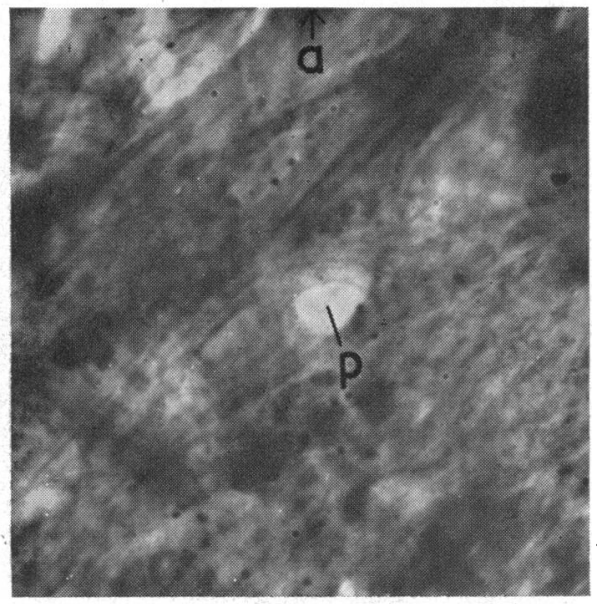

FIG. 9.-Flat preparation of trabeculae viewed from external aspect, i.e. inner wall of Schlemm's canal. Note stoma ( $p)$. ( $a=$ anterior). $\times 735$.

FIG. 10.-Photomicrograph of tilted frontal section showing stoma $(p)$ in inner wall of Schlemm's canal $(s) . \quad \times 350$.

The endothelial layer is very thin and is probably a prolongation of the corneal endothelium, but the cells are flat and their limits in-

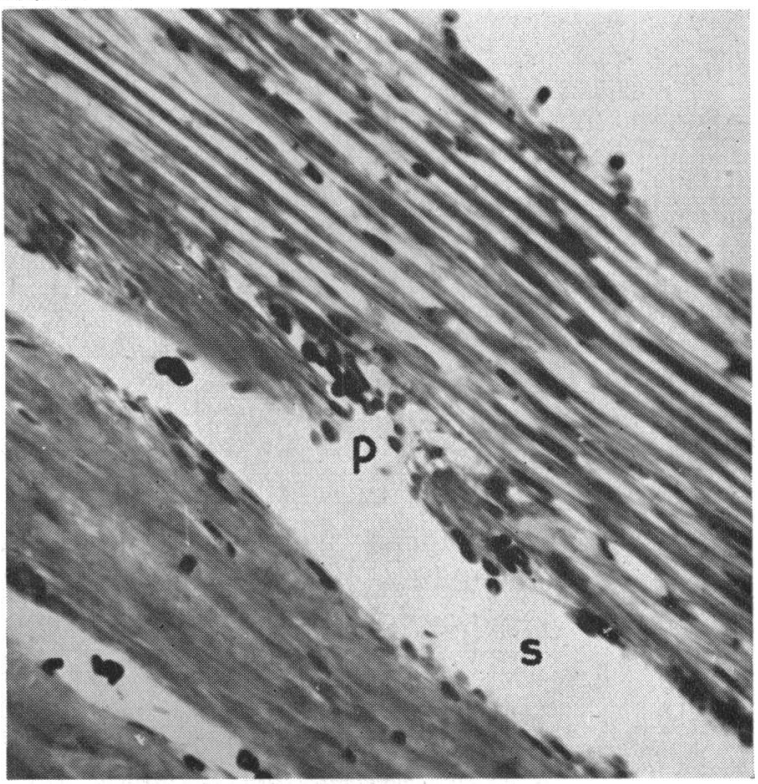
discernible.

The underlying glass membrane is probably a product of the endothelial layer, being comparable to Descemet's membrane, but it does not appear to be merely an extension of Descemet's membrane, for with periodic acidSchiff staining the corneal glass membrane stains an intense red whereas the trabecular glass membrane stains a delicate pink.

The elastic layer, according to Salzmann, lies immediately beneath the glass membrane and the elastic fibres are visible in the form of dots. It is true that most of them are on the surface of the connective tissue core, but we also found them very frequently within the substance of the core itself. Elastic fibres appear to be more numerous in the posterior as opposed to the anterior region of the trabecular meshwork.

The inner core itself is not homogeneous, but shows granularity (in 95 per 


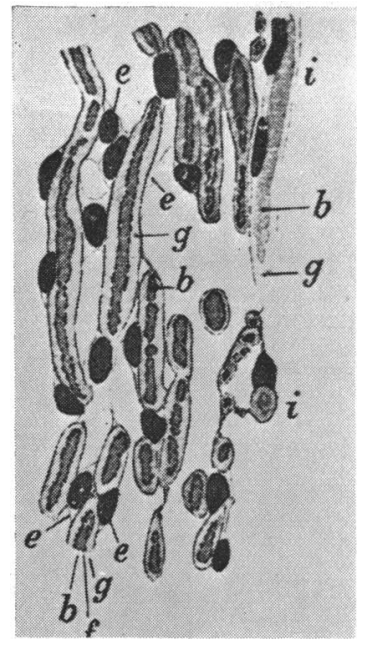

FIG. 11.-Drawing of trabeculae (after Salzmann, 1912). $b=$ collagen; $e=$ endothelium; $f=$ elastic fibres; $g=$ glass membrane; $i=$ uveal trabeculae.

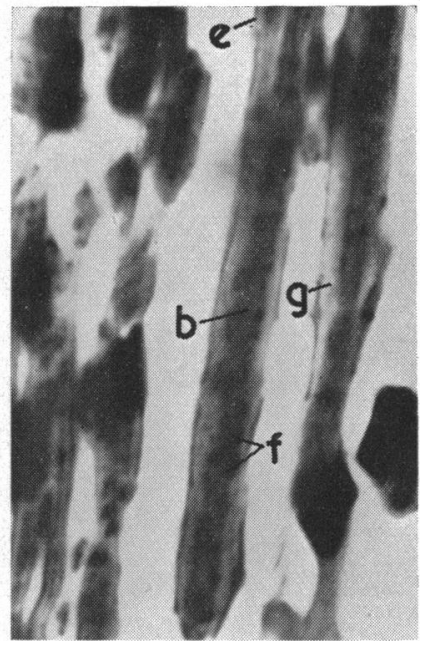

Fig. 12.-Photomicrograph of antero-posterior section of trabeculae. $e=$ endothelial layer; $g=$ glass membrane; $f=$ elastic fibres (cut ends appear as dots); $b=$ connective tissue core. $\times 1400$.

cent. of the eyes we examined), which is probably due to a cross-sectional view of the numerous fine circumferential fibrils described in the flat preparation. Indeed, this granularity is never seen in tilted frontal sections, which are parallel to the direction of these fibrils.

In antero-posterior sections, the different planes of the meshwork are seen to be separated from one another by gaps of variable size, which may be bridged by slender endothelial strands or by fully formed trabecular fibres.

(ii) Tilted Frontal Sections.-These also clearly show the four different layers of the trabeculae, but the elastic fibres are now seen as black lines running in the direction of the trabecular fibres. They are sometimes in two

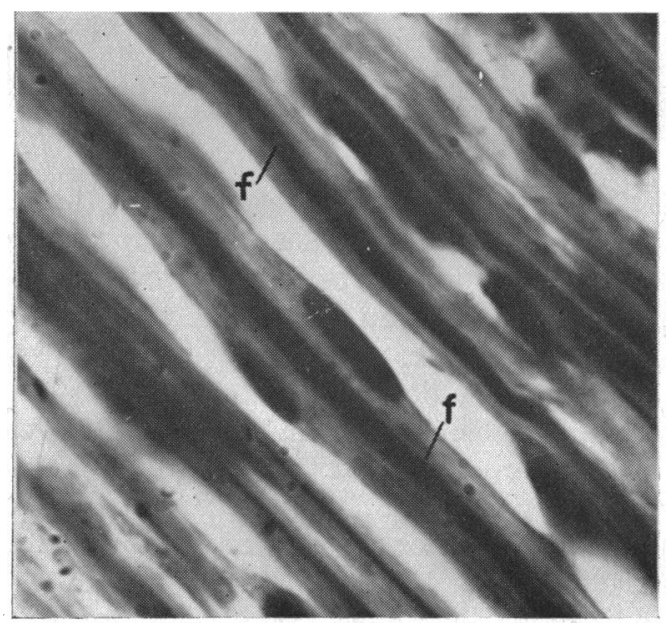
parallel lines, as described by Salzmann, but they often appear to be distributed irregularly and may be seen in the connective tissue core (Fig. 13).

FIG. 13.- Tilted frontal section of trabeculae. Elastic tissue $(f)$ now appears as long lines, confirming its predominantly circumferential orientation. $\times 1400$. 
(iii) Flat Sections.-Here it is more difficult to distinguish between the different layers. As noted by Salzmann, the glass membrane is thinner on the edges of the trabecular bands than upon the surface. Elastic fibres are easily shown by the usual special stains (Weigert, resorcin, fuchsin-orcein, Verhoeff) which all give equally reliable results (Fig. 14).

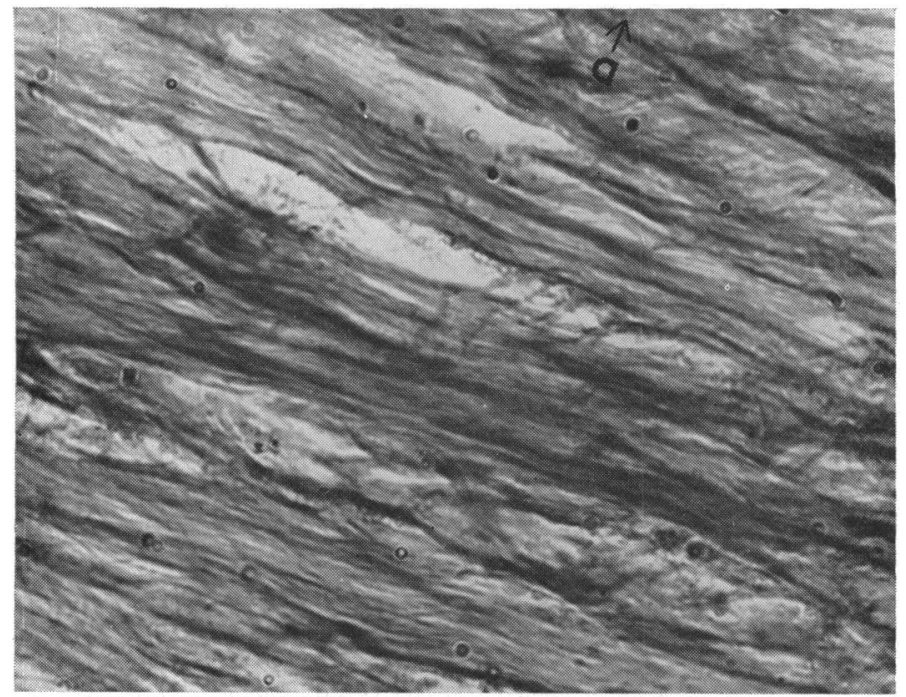

FIG. 14.-Flat section of trabeculae. Predominant direction of fibres is circumferential ( $a=$ anterior). $\times 433$.

(iv) Flat Preparations.-Stained with polychrome methylene blue, these show metachromasia in the internal layer of the trabecular bands. By changing the incidence of the light, the glass membrane can be made visible in the form of a bright translucent tissue on the edge of the bands. Numerous darkly staining fibres give the trabeculae a felty appearance; these are either elastic fibres or connective tissue fibrils (Fig. 15).

Fig. 15.-Flat preparation of trabeculae. Numerous fibres disposed mainly circumferentially $(a=$ anterior $) . \quad \times 433$.

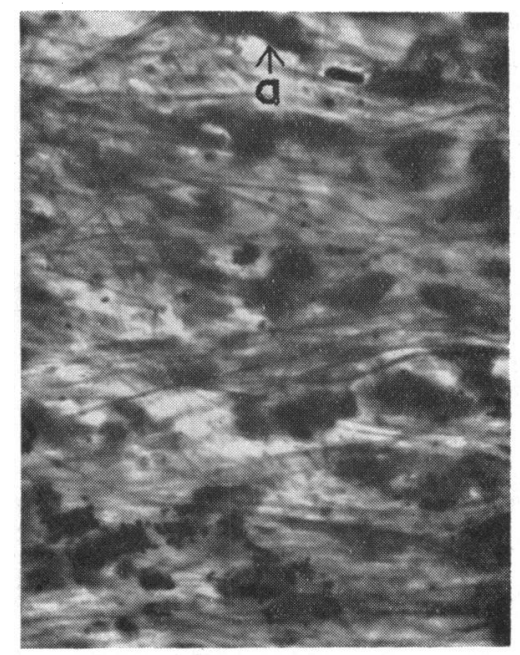


(v) Phase-Contrast Microscopy.-This was not of great value in this study, but was useful for demonstrating elastic fibres, which are readily visible in unstained antero-posterior and tilted frontal sections (Fig. 16).

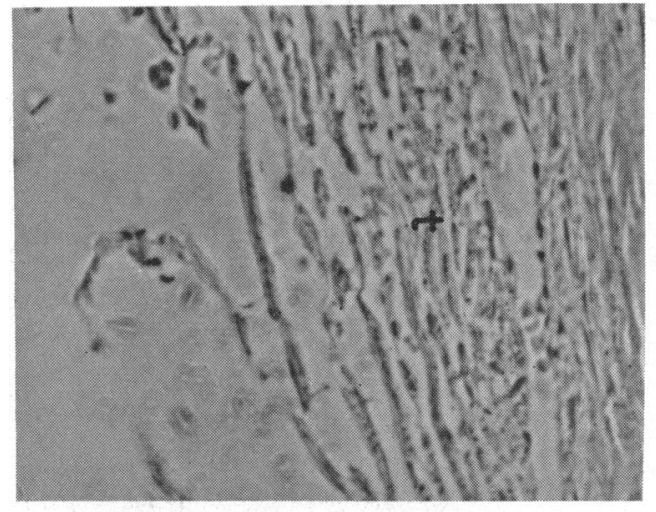

Fig. 16.-Phase-contrast microscopy of trabeculae $(t) . \quad \times 340$.

(d) Dimensions of Trabecular Fibres and Pores.-The following were the approximate dimensions found in studies with the micrometer eye-piece in antero-posterior sections.

\begin{tabular}{|c|c|c|}
\hline Total thickness of meshwork near sc & $\mathrm{ll} \mathrm{sp \imath}$ & $120 \mu$ \\
\hline Thickness of each trabecular fibre... & $\ldots$ & $5 \mu$ \\
\hline Space between trabecular layers: ... & $\ldots$ & $\ldots 0-5 \mu$ \\
\hline Diameter of trabecular holes: & $\ldots$ & ... $2-12 \mu$ (very variable) \\
\hline
\end{tabular}

Any of the above dimensions may vary within wide limits in the same eye or in various parts of the same trabecular fibre. In view of this fact it was decided not to extend this study to sections in other planes. It was clear from observations of the flat preparations that the size of the holes in the trabeculae, and the width of the trabecular bands delineating them, were of such variable dimensions as to render a representative numerical assessment impracticable. Thus, in our view, it is not possible to estimate deviations from the normal by measurements of these structures.

(e) Enumeration of Pores.-The object of this study was to establish whether the average number of pores in the wall of the canal differed markedly from the average number in each lamina. It was thought that if the average number in each lamina were found to be similar to the average number in the wall of the canal it could reasonably be taken as slight additional evidence in favour of the existence of through and through channels.

The gaps, or pores, in each lamina of the meshwork, including that forming the inner wall of Schlemm's canal, were counted in antero-posterior sections of 34 eyes. The area to be examined was viewed through a rectangular aperture which allowed a view of the anterior portion of the canal and of the trabeculae between this and the anterior chamber. 
It was found that the average pore count in this limited area of the canal's inner wall was 0.5 per section, and the average number for each trabecular lamina proved to be $0 \cdot 59$. It was concluded, therefore, that the number of pores in each layer, including the wall of the canal, is approximately the same. In addition it was noted that the pores tend to become.smaller as the canal is approached.

(f) Communication between Anterior Chamber and Canal of Schlemm.Numerous sections were seen in which a pore in the inner wall of Schlemm's canal opened freely into an inter-trabecular space (Fig. 17).

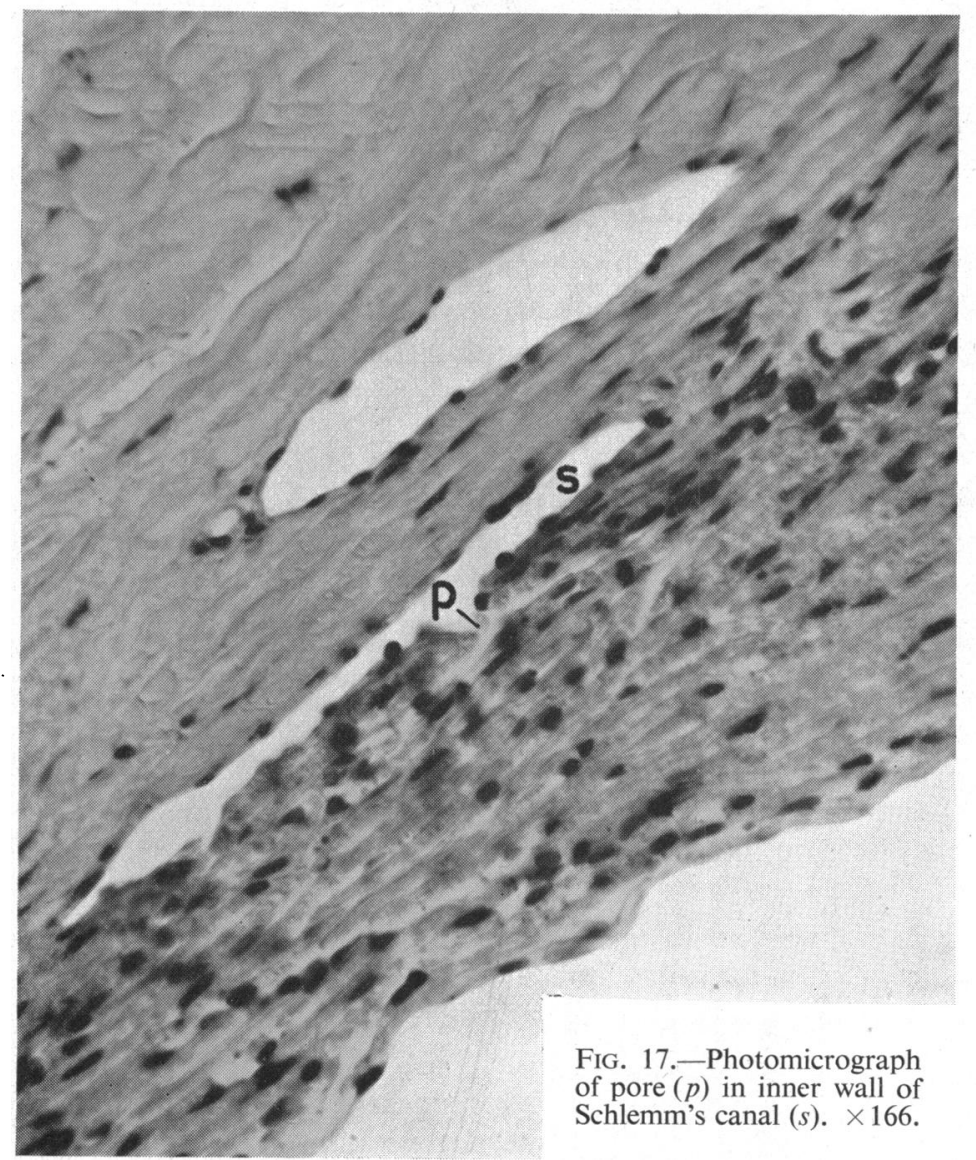

Furthermore, in other cases, a few red cells were seen lying within the canal in a random fashion, while adjacent to them, in the nearest intertrabecular space, were seen other red cells, so orientated as to suggest that they were in fact trapped between two laminae of the meshwork (Fig. 18, overleaf). This appearance was seen in eyes in which no blood was present in the anterior chamber, and it therefore seems clear that the red cells must have gained access to the inter-trabecular spaces from the canal of Schlemm. 


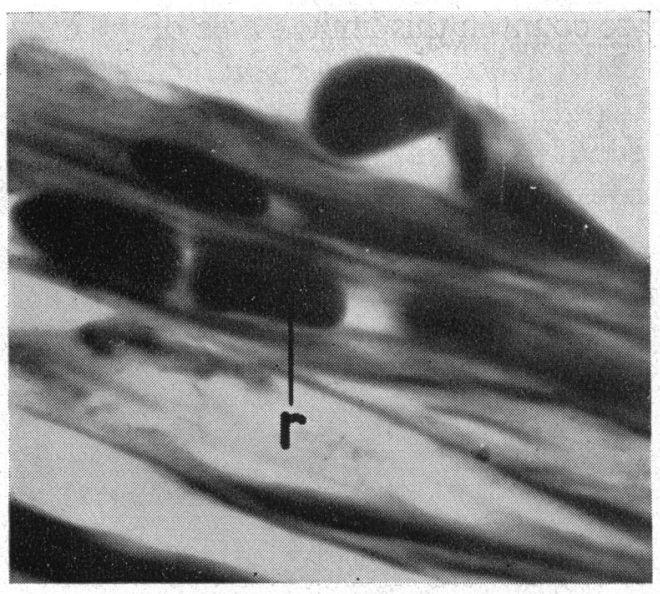

Fig. 18.-Tilted frontal section. Erythrocyte $(r)$ present between trabecular laminae. $\times 2100$.

Serial sections of a normal post-mortem eye confirmed the impression of the existence of channels leading from the canal of Schlemm into the intertrabecular spaces. Broken ends of epithelium suggesting rupture of a previously intact lining were never found in the sections examined. Attempts to produce macroscopical casts of these channels with Neoprene gave inconclusive results.

(g) Variations in Histological Appearance of Meshwork.-The classical picture of regularly disposed trabeculae separating an oval canal of Schlemm from the anterior chamben is frequently not found in routine sections embedded in celloidin and stained with haematoxylin and eosin.

(i) The walls of Schlemm's canal may be applied to one another to a variable extent and occasionally the canal is completely obliterated. This appearance may be interpreted or misinterpreted as "adhesions".

(ii) Individual trabeculae are often closely applied to one another and some sections show no inter-trabecular spaces, the meshwork appearing as a solid mass.

(iii) A granular appearance within the cores of individual trabeculae can result from cross-section of the numerous fine fibrils within the trabecular fibres, such appearances not being evident in parallel or tilted frontal sections (Figs 19 and 20, opposite).

(iv) Staining of the trabeculae is frequently not uniform, and pale areas, which might or might not indicate degeneration of the meshwork, may be evident in the region adjacent to Schlemm's canal.

(v) The trabeculae may present a blurred and tattered appearance, as though they had been torn across rather than cleanly sectioned. In such specimens the appreciation of the trabecular architecture is entirely lost.

(vi) Sections not passing through the pupil cut the trabecular meshwork in a plane which is neither at right angles to the plane of the laminae nor parallel with them, but passes through them obliquely. 
Fig. 19.-Pseudo-“ granularity" of trabeculae. Section taken in antero-posterior plane shows cut ends of elastic fibrils as dots $(f): \quad \times 1575$.

(Cf. Fig. 20).
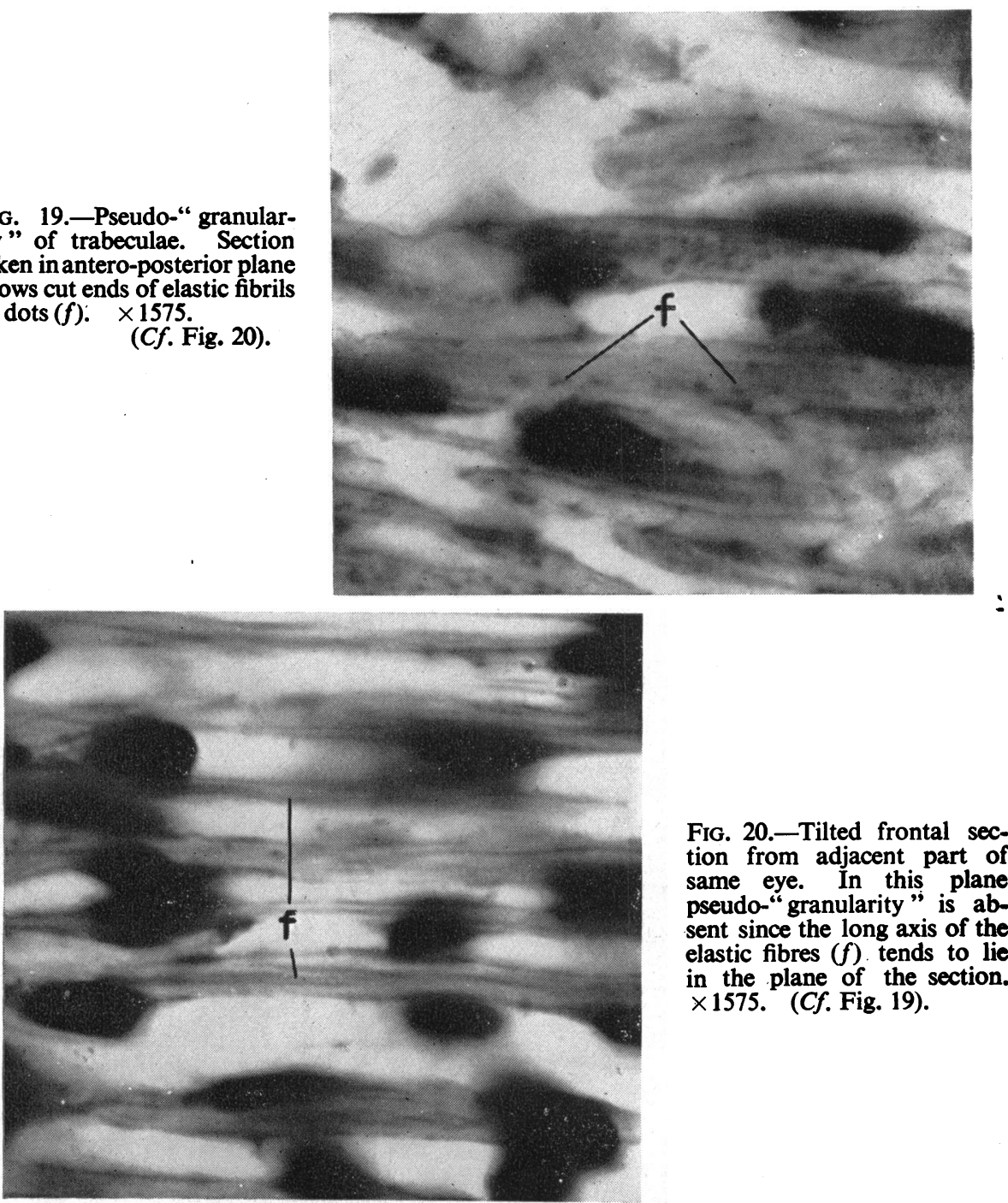

Fig. 20.-Tilted frontal section from adjacent part of same eye. In this plane pseudo-" granularity" is absent since the long axis of the elastic fibres $(f)$. tends to lie in the plane of the section. $\times$ 1575. (Cf. Fig. 19).

Such sections produce a curious artefact, in that the individual elastic fibres of the trabeculae appear to be inclined at an angle to the canal, being directed towards the anterior chamber as they pass forward. The explanation of these appearances may be seen in Fig. 21 (overleaf).

In Section $a$, passing through the meshwork at right angles to the planes of the laminae, the cut ends of the elastic fibres are seen as dots; in Section $c$, passing through the meshwork parallel to the planes of the laminae, the fibres appear as long lines.

In Section $b$, passing through the meshwork at an intermediate angle, the elastic fibres are seen as short lines, their apparent length being related to the thickness of the section. Furthermore, because the plane of the trabecular meshwork is neither antero-posterior nor coronal, but is itself inclined, the short lines seen in Section $b$ are also inclined. This appearance is illustrated in Fig. 22 (overleaf) and may be demonstrated constantly in sections in Plane $b$. 


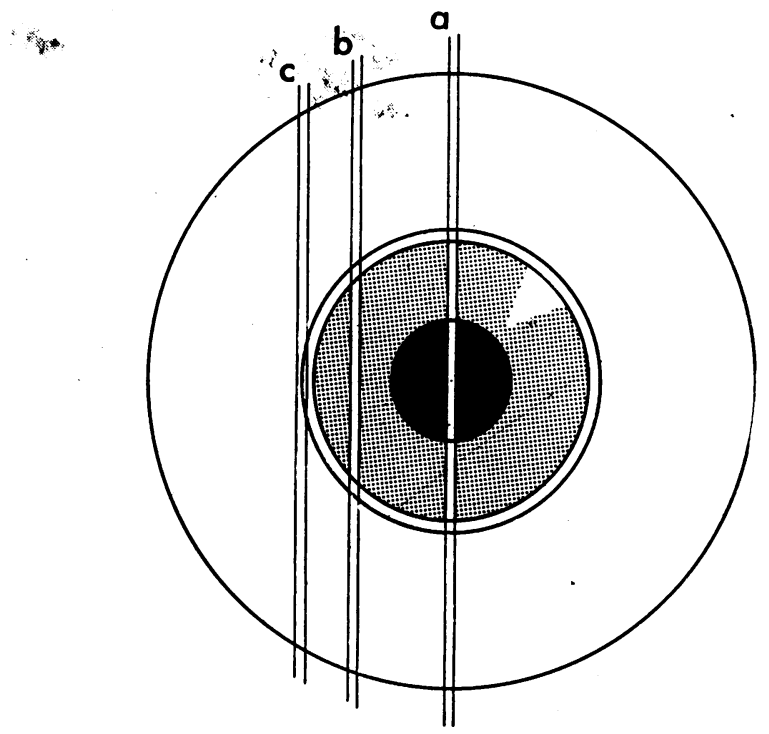

FIG. 21.-Three possibilities in antero-posterior sections: (a) Conventional view; (b) Artefact of trabecular obliquity as shown in Fig. 22; (c) Flat section of small part of trabeculae.

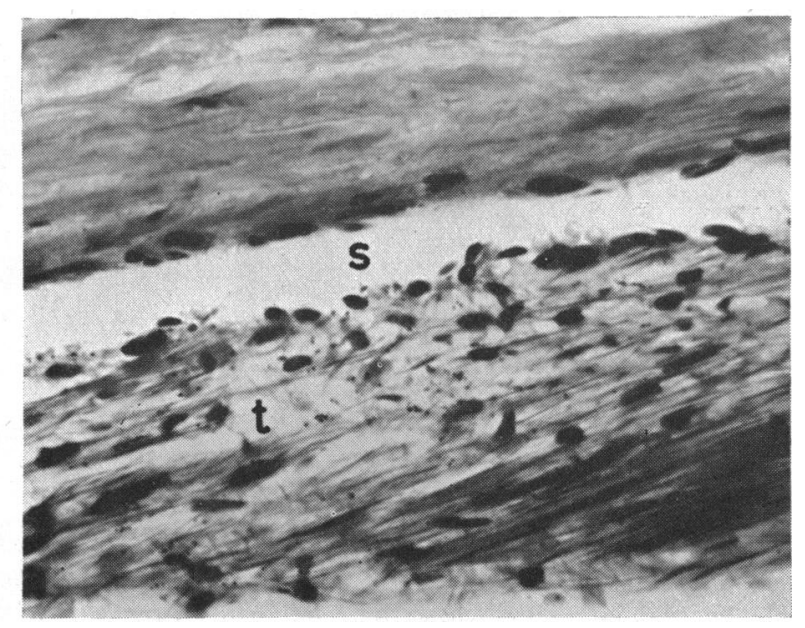

Fig. 22.-Oblique appearance of normal trabeculae as seen in an antero-posterior section made well away from the midline (Plane $b$, Fig. 21). $s=$ Schlemm's canal; $t=$ trabeculae. $\times 382$.

The results of a search for such artefacts in 148 eyes are summarized in the Table (opposite).

It is here apparent that granularity of the trabeculae in antero-posterior sections is almost invariable (for the reasons already given), and that adhesions or apparent adhesions of the walls of Schlemm's canal occur in about 25 per cent. of sections irrespective of age. (In this investigation " adhesions" were recorded if the walls were apposed in more than one-third of the antero-posterior dimensions of the canal. In fact, most of the examples quoted had apparent complete obliteration of the canal). 
TABLE I

HISTOLOGICAL ARTEFACTS IN THE REGION OF THE FILTRATION ANGLE

\begin{tabular}{|c|c|c|c|c|}
\hline \multicolumn{2}{|l|}{ Artefacts } & $\begin{array}{l}\text { Number of } \\
\text { Angles } \\
\text { Observed }\end{array}$ & $\begin{array}{l}\text { Number of } \\
\text { Angles } \\
\text { Affected }\end{array}$ & $\begin{array}{l}\text { Percentage } \\
\text { Affected }\end{array}$ \\
\hline \multicolumn{2}{|c|}{ Granularity of Trabeculae (all ages) } & 296 & 282 & 95 \\
\hline $\begin{array}{l}\text { Blurring of } \\
\text { (all ages) }\end{array}$ & Architecture & 281 & 115 & 39 \\
\hline \multicolumn{2}{|c|}{ Apposition of Trabecular Layers ... } & 281 & 51 & 17 \\
\hline \multirow{9}{*}{$\begin{array}{c}\text { " Adhesions" of Canal Walls } \\
\text { (Age in years) }\end{array}$} & $0-9$ & 6 & 3 & 50 \\
\hline & $10-19$ & $\mathbf{0}$ & 0 & - \\
\hline & $20-29$ & 4 & 2 & 50 \\
\hline & $30-39$ & 8 & 5 & 63 \\
\hline & $40-49$ & 58 & 16 & 26 \\
\hline & $50-59$ & 72 & 17 & 23 \\
\hline & $60-69$ & 78 & 20 & $25 \cdot 5$ \\
\hline & $70-79$ & 34 & 8 & $23 \cdot 5$ \\
\hline & $80-89$ & 4 & 2 & 50 \\
\hline
\end{tabular}

The figures are expressed as "angles" and not as eyes, since in several eyes one side of the section was damaged and hence unsuitable for observation. In the section on "Adhesions" of Canal Walls it is apparent that no
significant difference exists in the percentage affected between the ages 40-79. Figures for the other decades are too small to be significant.

(h) Staining Reactions.-With haematoxylin and eosin a fairly good differentiation of the trabecular structure is obtained in varying shades of red. With van Gieson's method, the inner core is pink, whereas the glass membrane is yellow. Masson's technique (ponceau-fuchsin and light green) shows a green inner core and a bright green glass membrane. The addition of Verhoeff's elastic stain shows the elastic fibres very well, and also stains the endothelium so that the four superimposed layers are clearly visible.

PAS stains Descemet's membrane dark red and the trabecular fibres pink, but does not differentiate the successive layers inside the fibres.

Metachromatic staining on flat preparations has not been always obtained with polychrome methylene blue and the reason for this inconstancy is not understood. On the other hand, with weak concentrations of toluidine blue, metachromasia in the inner core of the trabeculae was constantly found. No metachromasia was found in any section which had previously been treated with hyaluronidase.

\section{Discussion}

Consideration of the evidence in the literature together with our own observations has enabled us to reach certain conclusions with regard to con- 
troversial opinions about the anatomy of the trabecular meshwork and to enumerate varied histological appearances due to artefact.

\section{ANATOMiCal CONSIDERATIONS}

With regard to the limits, general shape, and histology of the trabeculae, our findings largely confirm the early classical descriptions and differ in only a few minor details which have already been described. However, the simultaneous use of sections in three perpendicular planes, together with flat preparations, has given a more accurate view of the general orientation of the trabecular fibres; indeed, the establishment of their circular distribution is one of the principal points to emerge from this work, for it is possible that this arrangement is a significant mechanical factor in the normal and abnormal function of the meshwork. Indeed, the concept that the trabecular meshwork functions simply as a filter has little to commend it, since in health there is nothing in the aqueous humour requiring filtration, and in disease it would probably be better if such solid material as may be present should be allowed to escape. It seems more likely that the meshwork plays some part in regulating the aqueous outflow, for the anatomical arrangement of the trabeculae suggests that the perforated elastic laminae may automatically alter their position relative to one another in response to pressure differences on either side of the meshwork, or with varying degrees of tension in the ciliary muscle. Thus may arise variations in the porosity of the meshwork with a resultant influence on aqueous outflow.

With reference to the question of free communications between Schlemm's canal and the anterior chamber, we have not succeeded in providing any evidence more conclusive than that recently advanced by Dvorak-Theobald (1955), but our observations are in complete agreement with her findings. The great difficulty in deciding whether patent channels exist or not lies in the exclusion of possible artefacts, for the demonstration of such pathways through the meshwork, either by section or by injection, is always subject to the criticism that the openings may have arisen through mechanical rupture of the delicate endothelium in preparing the specimen. Several facts, however, favour the existence of such open channels:

(a) There seems no question that open communications exist on the inner surface of the meshwork and that all inter-trabecular spaces freely intercommunicate, so that it is only necessary to demonstrate that these spaces connect directly to the canal to conclude that open pathways are present through the whole meshwork.

(b) Patent channels passing out from the inner wall of Schlemm's canal into the adjacent trabecular spaces are constantly seen in serial sections, and, in addition, we have demonstrated them as oval or round stomata in flat preparations. Endothelial rupture at the edge of these gaps is never seen.

(c) We have shown that the number of pores in the trabecular lamellae is similar for each successive layer, including the inner wall of the canal. It is 
consequently probable that the meshwork layer which limits Schlemm's canal internally does not differ from the other layers.

We believe, therefore, that open pathways between the anterior chamber and Schlemm's canal do in fact exist, not in the sense of definite endotheliallined tubes regularly disposed, as suggested by the term " internal canals", but rather as tortuous communications wandering irregularly and obliquely through the meshwork, like channels through a sponge. The size and patency of these communications would appear to be governed by the size of the lamellar pores, which we have found to be of very variable dimensions, probably altering even further with the degree of tension in the lamellae, and by the width of the inter-trabecular spaces, which necessarily varies with the degree of compactness in the meshwork (Fig. 23).

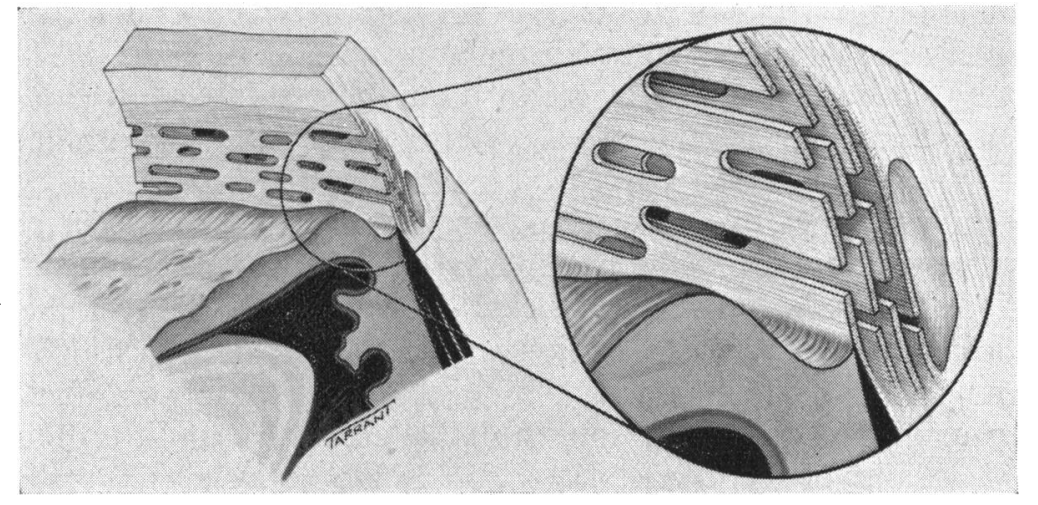

FIG. 23.-General arrangement of trabeculae.

One would not, therefore, expect much uniformity in the results of measuring pore-size by injecting particles into the anterior chamber, partly because of the irregularity of the channels themselves, partly because of the mechanical factors involved in compressing the meshwork, and partly because filtration is a highly complex process. A filter is not to be regarded simply as a mechanical sieve; many other factors, apart from particle-size in relation to pore-size, are concerned. Thus the electrical charge on the particle and on the filter, the effect of adsorption, the thickness of the filter, the nature of the suspending fluid, the concentration of particles, the possibility that larger particles may block the passage of smaller particles, and the temperature, pressure, and duration of filtration, may all influence the results to a considerable degree.

According to François and others(1955), the maximum particle size capable of traversing the meshwork is $2 \mu$; according to Huggert (1955) this size is $4.4 \mu$. It seems possible that under certain conditions red blood cells (average diameter 7.2 $\mu$ ) can find their way from Schlemm's canal into the trabeculae, where they are not infrequently seen in sections, and into the anterior chamber, where they have been observed gonioscopically. 
Having concluded that open channels are present and that they are not rigid tubes of set dimensions, but tortuous pathways of varying calibie, there is no difficulty in accepting the above findings as examples of the range of particle-size which may in differing circumstances pass through an undamaged meshwork.

\section{VARIATIONS IN NoRmal APPEARANCE}

We have previously enumerated the variations in normal appearances which may result from differences in methods of examination (p. 265). Since sections of the region of the angle in the healthy subject may present so many differing aspects, it is clear that great care should be taken before unusual or unfamiliar changes in this area are attributed to a pathological process.

In an anatomical study of the angle of the anterior chamber and the drainage channels in " normal" and glaucomatous eyes, Teng and others (1955) concluded that degenerative changes in the trabeculae, and in the canal of Schlemm itself and its efferent vessels together with endothelial proliferation, were a common occurrence in aged eyes, and were more marked in the eyes of three known cases of simple glaucoma studied by them. The type of degenerative changes seen were granularity of the collagen, degeneration of elastic fibres, hyalinization of endothelial cells, adhesions between the inner wall of Schlemm's canal, and distortion and obstruction of collector channels.

This report is of great interest in view of the decreased facility of the drainage of the aqueous humour in simple glaucoma, but it is difficult to assess the validity of their conclusions, for, as we have shown, very similar appearances of granularity, hyalinization, and "adhesions" may be attributed to artefact, and these possibilities do not appear to have been adequately considered by Teng, Paton, and Katzin, especially since the eyes in question were obtained from an eye bank and had been kept in saline for some days. The authors claimed that the possibility of artefact had been ruled out " by comparison with the most sensitive portion of the eye, the retina", but the nature of this comparison was not stated. Since one would expect considerable degenerative change in the retinae of eyes removed from the cadaver and stored in an eye bank, it is difficult to see how the authors were able to exclude artefact in this way. Furthermore, the admissibility of any one of their three cases of "wide-angle glaucoma" seems doubtful. Neither of the first two cases was authenticated, and in the third case the filtration angle was stated to have been closed in one eye and partially closed in the other-features which, in the absence of operation (and none is reported), almost certainly invalidate a diagnosis of wide-angle glaucoma.

On the other hand, we have no evidence that would contradict their conclusions, and their contention that the changes they describe are in fact pathological is supported by the rising incidence with age and by the fact that no degeneration was found in the anterior chamber angle in patients aged 20 years or less. Furthermore, we have not been able to produce these de- 
generative changes by leaving an eye in saline for several days. Their findings must therefore undoubtedly be accepted as a stimulus for further studies but should at present be regarded as unproven.

\section{SUMMARY}

It is suggested that recent trends in research on glaucoma necessitate a precise knowledge of the anatomy of the uveo-scleral trabecular meshwork. The literature on the subject is reviewed with regard to previous descriptions of the following features:

Limits and general shape of the meshwork from the histological and gonioscopical points of view,

Shape and orientation of the trabecular fibres and the histological constitution of each fibre,

Dimensions of the trabeculae,

Presence of free communications through the meshwork between the anterior chamber and Schlemm's canal,

Presence of nerves in the trabecular meshwork.

The present study consists of the examination of the trabecular meshwork of the human eye, by histological sections in three different planes and by flat preparations of fresh specimens. The material used included post-mortem specimens and eyes removed for malignant melanoma of the choroid.

The findings in the main confirm the work of previous authors:

The meshwork may be divided into an inner uveal part (containing outer and inner components) and an outer scleral part, but further definite subdivisions are not readily demonstrable.

The shape and orientation of the fibres appear to vary according to the plane of the section; antero-posterior sections show short rods, while circumferential sections show longer rods disposed circumferentially. It is concluded therefore, that the arrangement is that of circumferentially disposed flattened bands, each perforated by oval stomata, the antero-posterior sections giving cross sections of the bands and hence appearing as rods. This conception is supported by flat sections and flat preparations.

Our findings regarding the histological constitution of each band agree with previous work.

An attempt is made to measure the dimensions of the trabecular components, but the figures obtained vary considerably as between different eyes and different areas in the same eye.

The existence of pores in the inner wall of Schlemm's canal connecting with the inter-trabecular spaces is confirmed.

It is concluded that patent tortuous pathways existing in the trabecular meshwork enable communication between the anterior chamber and Schlemm's canal.

Variations in the normal histological appearance of the meshwork due to artefact are listed. 
No correlation was found between the histological appearances and the age of the patient.

\section{REFERENCES}

Asayama, J. (1901). v. Graefes Arch. Ophthal., 53, 113.

Bangerter, A., and GoldmanN, H. (1941). Ophthalmologica (Basel), 102, 321.

Beuningen, E. G. A. VAN (1949). v. Graefes Arch. Ophthal., 149, 637.

BRÜCKE, E. (1847). "Anatomische Beschreibung des menschlichen Augapfels". Reimer, Berlin.

Burian, H. M., Braley, A. E., and Allen, L. (1954). Trans. Amer. ophthal. Soc., 52, 390.

BusACCA, A. (1945). "Elements de gonioscopie normale, pathologique, et expérimentale". Tipografia Rossolillo, São Paulo.

De Lieto Vollaro, A. (1902). Arch. Ophtal., 22, 311.

Duke-Elder, S. (ed.). (1955). "Glaucoma. A Symposium organized by the Council for International Organizations of Medical Sciences". Blackwell, Oxford.

Dvorak-Theobald, G. (1934). Trans. Amer. ophthal. Soc., 32, 574. (1955). Amer. J. Ophthal., 39, No. 4, pt. 2, p. 65.

EISLER, P. (1930). "Kurzes Handbuch der Ophthalmologie", ed. F. Schieck and A. Brückner, vol. 1, p. 100. Springer, Berlin.

François, J. (1948). Bull. Soc. belge Ophtal., No. 88, p. 3.

, Neetens, A., and Collette, J. M. (1955). Amer. J. Ophthal., 40, 491.

FriedenWald, J. S. (1936). Arch. Ophthal. (Chicago), 16, 65.

HENDERSON, T. (1908). Trans. ophthal. Soc. U.K., 28, 47.

HoвBs, H. E. (1950). British Journal of Ophthalmology, 34, 489.

HUGGERT, A. (1954). Acta ophthal. (Kbh.), 32, 519. (1955). Ibid., 33, 271.

Iwanoff, A., and Rollett, A. (1869). v. Graefes Arch. Ophthal., 15, pt. 1, 17.

Kolmer, W. (1914). Anat. Anz., 47, 417.

Kronfeld, P. C., McGarRy, H. I., and Smith, H. E., (1942). Amer. J. Ophthal., $25,1163$.

LAUBER, H. (1936). In " Möllendorffs Handbuch der Mikroskopischen Anatomie des Menschen", vol. 3, pt. 2, p. $232 . \quad$ Springer, Berlin.

LEBer, T. (1895). v. Graefes Arch. Ophthal., 41, pt. 1, p. 235. (1903). In "Graefe-Saemisch Handbuch der gesamten Augenheilkunde", 2nd ed., band 2, abt. 2, kap. 11, p. 276. Engelmann, Leipzig.

LOEWENSTEIN, A. (1951). Ophthalmologica (Basel), 122, 257.

MagGiore, L. (1917). Ann. Ottal., 40, (n.s.l.), 317.

REDSLOB, E. (1939). In "Traité d'ophtalmologie", vol. 1, p. 556. Masson, Paris.

ROCHON-DUVIGNEAUd (1892). Arch. Ophtal., 12, 732. (1893). Ibid., 13, 20, 108.

Salzmann, M. (1912). "The Anatomy and Histology of the Human Eyeball ", trans. E.V.L. Brown, p. 41. Univ. Press, Chicago.

SchWALBE, G. (1870). Arch. mikr. Anat., 6, 261.

Sondermann, R. (1930). v. Graefes Arch. Ophthal., 124, 521.

Sugar, H. S. (1951). "The Glaucomas", p. 25. Mosby, St. Louis.

SWINDle, P. F. (1937). Arch. Ophthal. (Chicago), 17, 420.

Teng, C. C., Paton, R. T., and Katzin, H. M. (1955). Amer. J. Ophthal., 40, 619.

Teulières, M., and BeauvieuX, J. (1930). Arch. Ophtal., 47, 497.

Trantas, A. (1928). Ibid., 45, 617.

Troncoso, M. U. (1947). "A Treatise on Gonioscopy". Davis, Philadelphia.

Virchow, H. (1910). "Graefe-Saemisch Handbuch der gesamten Augenheilkunde ", 2nd ed., band 1, abt. 1, kap. 2, p. 281. Engelmann, Leipzig.

VRABEC, F. (1954). Ophthalmologica (Basel), 128, 359.

WALDEYER, W. (1874). "Graefe-Saemisch Handbuch der gesamten Augenheilkunde ", 1st ed., vol. 1, p. 169. Engelmann, Leipzig.

WolfF, E. (1954). " "The Anatomy of the Eye and Orbit ", 4th ed., p. 44 . Lewis, London. 